\title{
Context-Aware Digitalization - Adapting solution development to the organizational context of SMEs
}

\author{
Tiina Nokkala \\ Turku University of Applied \\ Sciences \\ Turku School of Economics, UTU \\ tiina.nokkala@turkuamk.fi
}

\author{
Altti Lagstedt \\ Haaga-Helia UAS \\ Turku School of Economics, UTU \\ altti.lagstedt@haaga-helia.fi
}

\author{
Robert Winter \\ Institute of Information Management \\ University of St. Gallen \\ robert.winter@unisg.ch
}

\begin{abstract}
In the context of digital transformation, it is mandatory for most organizations to conduct information systems development (ISD) projects as part of their digitalization and business development journey. One reason that many ISD projects fail is lack of knowledge about which ISD method (ISDM) is most suitable for the project at hand and how to adapt it to reflect the respective business development context. These problems especially occur in small and medium enterprises (SMEs), as they often lack specific methodological skills and project governance experience - so they cannot even manage ISD consultancies that promise to support them in their digital transformation. In this conceptual paper, we present the design of a method for selecting and using ISDM for SMEs. It considers both the context dependency and missing project governance skills of SMEs. The main components of the proposed method link the knowledge areas of business development and ISD: business context evaluation, ISDM selection and ISDM management.
\end{abstract}

\section{Introduction}

Currently, digitalization drives many organizations of all sizes to take initiatives to advance their business model, e.g. to develop, buy or modify information systems (IS) that support or enable the business and to implement them. However, many of these usually complex initiatives fail [see, e.g., 40], and an insufficient consideration of the organizational context can be one of the key reasons [27]. That is especially true with small and medium-sized enterprises (SMEs), as their nature and environment vary extremely from organization to organization $[7,20]$. Executing a digitalization initiative, e.g. developing and implementing an online booking system in a multioutlet barber shop is rather different issue than developing and implementing an internal follow-up system in a small marketing company, for instance. Almost the only thing SMEs have in common is their typical lack of digitalization professionals, and whereas not all SMEs really need customized or self-developed IS solutions, there is a huge potential for those who manage to create and utilize customized solutions to differentiate their business. However, well-executed digitalization initiatives have the potential to significantly improve SMEs' performance [7] in almost any field, and failure to conduct digitalization projects may result in competitive disadvantages [20].

Organizational and business development through developing new digital business and digitalizing existing business is almost always interwoven with information systems (IS) development. As a consequence, digitalization initiatives are usually realized, at least in part, through developing individual and/or customizing standardized software. Even if offthe-shelf standardized software solutions need to be customized and introduced, outside developers are often brought in, as most SMEs do not have adequate competencies or ambitions. SMEs also have specific requirements for the developed product, due to their size and varying business contexts. A customized IS solution can enable differentiation of SMEs business, and thus these requirements cannot be passed - even if other IS components can be used "off-the-shelf". As a consequence, both information systems (IS) developers and buyers struggle with IS development (ISD) projects [41]. The buyers of ISD services often have little or no knowledge about suitable ISD methods (ISDM), and developers or consultants tend to select the method they know the best or the one that they use commonly [28]. In addition to the method selection aspect, the business context (e.g., stability of the business environment, maturity of business processes, stability and completeness of business requirements, prior experience) is often not sufficiently considered by the developers or consultants, and the existing software 
development methods may not have fundamental context adaptation mechanisms. Thus, the ISD project is compromised in the very beginning through a poor and context-unaware selection of ISDM and later through the inefficient use of the selected ISDM.

Even if the capability gap is addressed by bringing in an IS developer and the context dependency is known to both parties, some problems persist. In ISD projects with a specific ISDM, IS developers have often regarded the value of the ISDM as low, and the ISDM may therefore have been modified and/or partially ignored [19, 34, 44]. If the value of the ISDM is regarded as low (i.e., the selected ISDM does not appear to suit the needs of the ISD project), it is easy to abandon that ISDM. In addition, it seems that developers and consultants, especially those specialized in serving SMEs, are sometimes not familiar with all relevant standards, models, methods and practices to produce trustworthy and secure software for the business needs in question [30, 31]. That, together with the fact that not all necessary modifications have been designed and implemented, leads to ill-fitting IS and consequently to distorted processes. The rising complexity of not only the IS but also the environments in which they are used, creates even additional requirements for ISDMs. Because of all this, it is essential that the ISDM selection and application suit the development project in question.

There is a gap in the research on selecting ISDM; some ISDM selection models and research exist, but it seems that they are more or less outdated [17]. Especially there is not much knowledge available on the specific ISDM selection challenges within SMEs. On the top level, ISDMs can be grouped into changedriven and plan-driven methods [37], and the first phase is to select between these. However, our aim in this paper is not to compare the ISDMs or make judgements between them, but to provide SMEsuitable tools for comparing ISDMs and other standards, and to make selections between them based on the respective business development context and other important factors. That is to say, our focus is on the management level, not on the development level. Since SMEs only rarely have capabilities to make these selections by themselves [22], we claim that they need lightweight support tools for the method selectionthat is, on the management level.

This research and the development of this type of lightweight selection method for ISDM is needed because SMEs have been neglected in digitalization studies [7]. At the same time, SMEs are lagging in digitalization compared to larger organizations $[6,7$, 22]. Theory-based solutions that improve the success rate of digitalization initiatives and IS development projects are needed. By including context-awareness in the selection and considering not only the project characteristics, but also the business environment and the overall dynamics of the project and development group, the solution is more comprehensive than the currently existing ones. The proposed lightweight method should be equally useful for the SMEs, their consultants and IS developers using it, as the meticulously selected ISDM enables value creation better than just selecting an ISDM without appropriate analysis and/or using it carelessly.

In this conceptual paper, we integrate the knowledge areas of digital business development and IS development. We present a preliminary conceptual model for selecting the ISDM in various business contexts and for appropriately utilizing and managing the selected method. The presented model consists of three phases: (1) the business context evaluation model, (2) the ISDM selection model and (3) the ISDM management model. These three components lay the groundwork for further development by conceptualizing business-aware ISDM selection. By presenting the conceptual model, we answer the following research question:

RQ: "How can ISDM decision-making be supported for SMEs”

Section 2 summarizes the theoretical background, including SME digitalization and its challenges. In Section 3 we present our proposed method and its features, and in Section 4 we outline the initial evidence supporting our method. In Section 5 we discuss the resulting method and make conclusions.

\section{Theoretical background}

To understand the current situation of SMEs, it is important to discuss the digitalization needs and challenges of SMEs. In addition to this overall approach, we consider in more detail method selectionrelated approaches of ISD, approaches to analyzing the development context, and existing work related to development method selection and method engineering. The purpose is to have a clear path from overall needs for SME digitalization to context-specific development method selection.

\subsection{Digitalization in SMEs}

Digitalization concerns all kinds of organizations and even the business areas normally not considered IT-oriented are facing the need to digitalize their processes [3, 6]. Digitalization is not just a managerial fad; as Bharadwaj et al. [3] pointed out, there are several reasons why digitalization is highly relevant for organizations. According to them, digital technologies enable global connectivity and new kinds of business strategies, they make reacting to turbulent situations 
possible and reshape social relationships (both consumer and enterprise level); work can be carried out without restrictions of time and distance; products and services increasingly have embedded digital technologies; and reduced component and service prices makes the change cheaper all the time [3]. All these make cross-boundary industrial disruptions possible [3]. Clearly organizations are not able to control (all) these reasons, and they are forced to react and adapt to the changing situation no matter whether they want it or not. In addition, the more the organizations digitalize, the more pressure they exert on lagging organizations.

However, it seems that the SMEs are not keeping the same pace of digitalization as large companies $[6$, $7,22]$. For example, according to Borg et al. [6], only about one-third of Swedish SMEs have individually developed (organization-specific) software solutions, whereas $55 \%$ of bigger organizations have individually developed software solutions (note that here they do not consider how the software is developed, i.e., if the developers are in-house or outsourced/contracted).

As SMEs have a remarkable role in the economy, the reasons why SMEs lag behind in digitalization are important to understand not only for SMEs, but for societies as well. As Bouwman et. al. [7] pointed out, digitalization studies focus mainly on large companies or high-tech start-ups; studies concentrating on digitalization and its challenges in SMEs are rare. So far, there are only some studies related to the topic. Canziani and Welsh [11] found that SMEs in the tourism business seem to have challenges in digitalization because they have restricted time and resources for digital change. Gruber [22] listed four main reasons why SMEs lag behind in digitalization. First, smaller companies often operate in business areas which have not so far needed rapid digitalization. Second, SMEs seem to lack knowledge, that is, they do not have personnel skilled enough to understand the implications of digitalization. Third, SMEs seem to proceed gradually (without big changes at once). Fourth, the financial possibilities for digitalization are limited [22]. Reflecting on the first reason, it really seems that digitalization is unevenly spread throughout industry sectors, with ICT, finance and insurance, and manufacturing being the leading sectors [6]. However, as already stated, digitalization is spreading to all business areas $[3,6]$, and this will cause pressure for SMEs whether they want digitalization or not [20]; there are no "protected" sectors anymore. As for the second and fourth of Gruber's reasons, we can see that they are related to some extent, but the lack of digitalization professionals is not only a financial issue. In small and medium-sized organizations there is not, nor has there been, a constant need for that kind of professionals; big development projects are rare, and in between big projects there is no need for such professionals. It is understandable that SMEs do not hire that kind of professionals (and the digitalization professionals are not eager to go SMEs where possibilities to use their skills are limited) [20]. The third reason Gruber [22] presents has a clear connection to the second and fourth reason: if SMEs do not have skilled resources, and especially not enough financial capability, they are forced to take very small steps [see, e.g., 11].

Since SMEs lack IS related resources, nor have many resources to allocate to development preparation, it is clear that high protocol, heavyweight models for ISDM selection are not to be used in SMEs. Often, if digitalizing organizations do not have the skills to make development method selections internally, they rely on IS suppliers [28]. This, on the other hand, has risks: since organizations digitalizing their businesses and IS suppliers have different business models and objectives for the project, the selection that appears to be optimal for a supplier is not automatically that for the client [28, 39, 42].

Thus, because of their lack of knowledge and skills, the SMEs are the ones who really need neutral guidance and help. Instead of accurate and exact - but heavyweight and resource-consuming - models, there is a clear need for low protocol, lightweight ISDM selection models.

\subsection{Information systems development methods and models}

Our aim is not to make judgements between the different kinds of ISDMs, but rather to provide SMEs tools for selecting the right combination of them. However, it is important to understand the main differences between the contemporarily used ISDMs to understand why the selection needs to be done and how the selection can be dependent on the business context.

A plethora of different ISDMs have been developed since the 1960s [32] and can be classified in several ways. We follow a control-based classification into plan-driven and change-driven methods [37]. During the last 20 years, there has been a clear paradigm shift from plan-driven dominance to change-driven dominance [43].

Plan-driven IS development follows the stage-gate model where one stage has to be completed before the next gets permission to start [see, e.g., Cooper 1990)]. This means that the whole project (objectives, resources, schedule) has to be planned before it is possible to start development [40]. However, even if all the definitions are done correctly, this does not guarantee success in IS development since 
circumstances might have changed [24]. Early mistakes that are found late are difficult (and costly) to resolve [40].

The change-driven approach is not problem-free, either. Planning and development are done in small iterative and incremental steps, and after each new step, a new IS version is released, the situation is reevaluated, and necessary changes are made to the objectives. The unplanned changes cause incoherencies in software architecture, which easily cumulate as a technical debt [14], causing more development (and maintenance) challenges in later phases. Furthermore, lack of vision, lack of shared understanding and constantly changing priorities cause the scope of development to “creep," and quality assurance becomes challenging [16, 37]. Since neither the plandriven nor the change-driven approach guarantees success for all cases, it is important to discuss (and select) a method for each particular case [29].

One alternative is to use a hybrid approach, in which certain components of plan-driven and changedriven development are combined [33, 38, 43]. It seems that the hybrid approach is widely adopted: twothirds (458 of 690) of the projects studied by Noll and Beecham [38] combine agile and traditional (plandriven) methods. As Marinho et al. [33] point out, if the hybrid approach is seen as a possible solution to adapt methods to a development situation, there should be a suitable strategy to configure it for each specific case.

\subsection{Business digitalization project context evaluation}

In their analysis of very large ISD projects, Winter at al. [47] identified several factors that explained, even in the presence of proper project management practices, why the projects failed. Their findings confirm certain factors that cannot be directly influenced by project management but nevertheless have a significant impact on project performance [23]. Such factors therefore need to be considered in ISDM design. Kiselev et al. [27] designate these factors as "contextual factors" and propose an assessment instrument to analyze the relevant project context with regard to the factors presented in Table 1. Based on their empirically validated impact on ISD project success, these factors should be considered when developing or customizing a development method.

As there currently exists no similar studies on SMEs and their contextual factors, we have adapted the factors recognized in larger projects to the SME context. Governing a digitalization project in a SME can be seen very similar to the larger digitalization, only the scale is smaller. The roles in the project, e.g. the project sponsor, the project manager and IS developer exist in the projects of all sizes. In SMEs, however, they are often incorporated in other roles, such as business owner or business manager, and thus the actors are often less skilled in project management in general, and in ISD projects in particular.

Table 1. Contextual factors according to Kiselev et al. [27]

\begin{tabular}{|l|l|}
\hline $\begin{array}{l}\text { Terrain, the technological } \\
\text { and conceptual territory an } \\
\text { organization enters through a } \\
\text { project, measured by: }\end{array}$ & $\begin{array}{l}\text { a) Experience with similar } \\
\text { project or solution } \\
\text { b) Existence of standard } \\
\text { solution in use } \\
\text { c) Sufficiency of existing } \\
\text { infrastructure } \\
\text { d) Experience with similar } \\
\text { organizational changes }\end{array}$ \\
\hline $\begin{array}{l}\text { Dynamics, measured by the } \\
\text { speed and ambiguity of: }\end{array}$ & $\begin{array}{l}\text { a) Technological } \\
\text { environment } \\
\text { b) Organizational } \\
\text { environment } \\
\text { c) Political environment } \\
\text { d) Legal environment } \\
\text { e) User demands }\end{array}$ \\
\hline $\begin{array}{l}\text { Complexity (both systemic } \\
\text { and organizational), } \\
\text { measured by: }\end{array}$ & $\begin{array}{l}\text { a) Amount of relevant } \\
\text { peripheral technical systems } \\
\text { and interfaces }\end{array}$ \\
$\begin{array}{l}\text { b) Complexity of system } \\
\text { architecture } \\
\text { c) Stakeholder heterogeneity } \\
\text { and independent progression } \\
\text { of a project, measured by: }\end{array}$ & $\begin{array}{l}\text { a) Domplexity of } \\
\text { organizational structures and } \\
\text { processes }\end{array}$ \\
\hline $\begin{array}{l}\text { Commitment, Budgeting cycles } \\
\text { standing and respect of } \\
\text { organization, measured by: }\end{array}$ & $\begin{array}{l}\text { a) Management commitment } \\
\text { b) Employee commitment }\end{array}$ \\
\hline
\end{tabular}

\subsection{ISDM selection}

The most common approach seems to be an ISDM selection based on project complexity and uncertainty [see, e.g., 10]. However, this approach has been criticized, and it has been questioned whether complexity and uncertainty are really the correct independent factors [25].

Among the more complex models, probably the most widely known is Boehm and Turner's [5] fivedimensional model, where the selection between agile and plan-driven methods is based on dynamism, culture, size, criticality and personnel of the ISD project. This model also has its challenges - for instance, dynamism is measured in changes/month, which is only possible to measure after the project is 
started and the method selected [29]. There are also other factors, mainly project-specific factors focused on ISDM selection models [see, e.g., 1, 24, 44].

Although several different kinds of ISDM selection models have been presented in recent decades, they have not been used widely, and critics maintain that the arguments behind these models are more or less outdated [17]. A common feature of the earlier models seems to be the assumption that business context characteristics have little or no effect on ISDM selection [29], despite the fact that, according to Dahlberg and Kivijarvi [15], factors outside the IS project domain are the most important determinants for IS project performance.

Lagstedt and Dahlberg [29] present an ISDM selection model that takes the business context of the ISD project into account (see Figure 1). Although their model is rather simplified (e.g., it does not discuss hybrid methods at all), it clearly describes how to consider project- and context-specific uncertainties. With its connectivity to the business context and business development models, we see it as a useful starting point for further development. It can also produce a metamodel for the development of a utilization model that can be adapted to the case at hand in a systematic form.

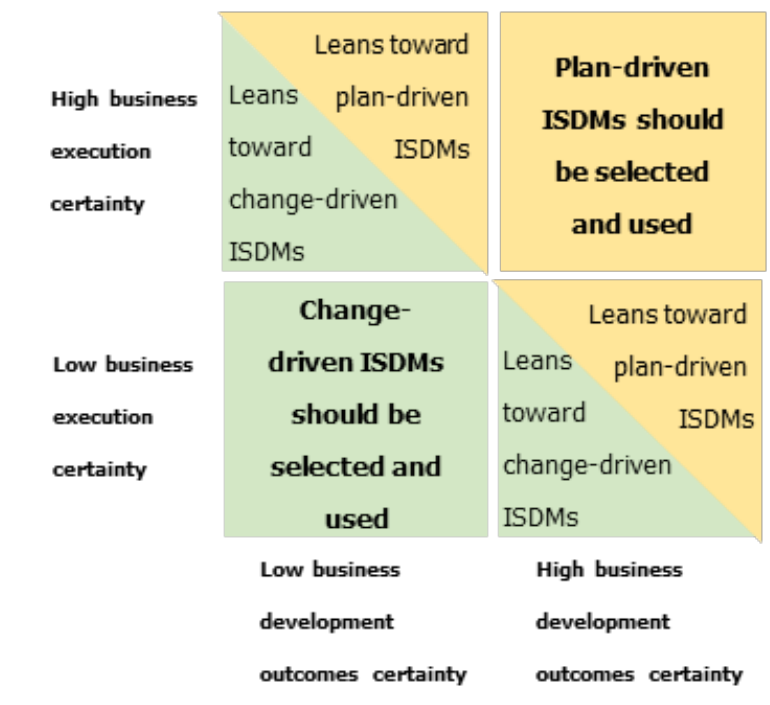

Figure 1. Lagstedt and Dahlberg (2018) model

\subsection{Situational method engineering}

Method engineering (ME) as a discipline primarily aims at the systematic construction of methods supporting the development of software artifacts [8]. Situational ME enhances the utility of ME by offering a mechanism that composes method modules or configures a base method so that the resulting method is adapted to a specific problem context and specific solution objectives [9]. As a consequence, a situated development method can be constructed for every specific case [26, 36]. Regardless of whether situational $\mathrm{ME}$ is based on composition or configuration, it always requires (a) a generic method foundation with built-in adaptation options, (b) knowledge about how different problem contexts and/or solution objectives influence method adaptation and (c) knowledge about the context and solution objectives for the problem at hand [46].

Aspect (c) requires understanding which contingencies are relevant for the respective problem class (e.g., ISDM selection for SME) and which general problem classes can be observed in the real world (e.g., integration of different mobile sales/service channels in small retail companies) [9]. Based on such empirical problem/objective clustering, existing problem-solving knowledge can then be used to determine how a general solution method (aspect a) should be systematically adapted (aspect b) [46]. For example, a generic collection of ISDM fragments/chunks (from both plan-driven and changedriven approaches) needs to be investigated to determine which combinations are useful in which problem classes. Based on the analysis, general configuration or composition rules can be derived.

While situational ME appears to provide a suitable conceptual foundation for context-aware ISDM selection, it requires considerable effort to create and maintain the adaptable method base (aspect a) and to sufficiently understand the problem context and solution objective contingencies of ISDM selection in the highly varied domain of SMEs. Thus, our aim in using situational ME as a basis for ISDM selection method is to use its features as checklists and simple rules that provide guidance for the development route.

\subsection{Technological rules}

A simplified way to adapt ISDMs to specific problem context characteristics and solution objectives is to base the adaptation on technological rules [2]. In their simplest form, technological rules can be represented as quadruples associating a specific context, an intervention type, a generative mechanism, and an outcome (CIMO pattern) [18]. For example, the analysis of our ISDM selection problem could confirm that:

- whenever the SME is aiming to support an innovative business process (which has not been supported so far by its business software) (context)

- choosing an agile approach and a local developer (intervention) 
- leads to higher alignment of desired and delivered business process software support (outcome)

- because constant interactions and small increments support mutual understanding between developer and SME and fast detection of misalignment in an unknown application domain (mechanism).

A different exemplary technological rule would indicate that:

- whenever the SME is aiming to increase the degree of automation and/or integration of an existing business process (context)

- choosing a traditional development approach (intervention)

- leads to higher efficiency of the software support optimization project (outcome)

- because existing knowledge (application landscape, successfully implemented integration services) and proven integration/automation capabilities can be reused and thus leveraged (mechanism).

While the concept of technological rules/CIMO patterns originates from the management discipline, the IS discipline has a similar discourse related to design principles whose structure has been proposed as a triple that associates certain material properties (in terms of form and function) of a system with certain actions of its users subject to certain boundary conditions (user group's characteristics or implementation settings) [12]. The similarity to CIMO patterns becomes even clearer with the recently proposed "anatomy of a design principle" [21] which is comprised of an aim (=intended outcome), a means of achieving such aim (=intervention), justificatory knowledge (=mechanism), and "context/boundary condition."

Design principles should be validated to be applicable to a class of artifacts - and hence also to a class of activities that instantiate a method. However, so far, design principles have been nearly exclusively proposed, and their design discussed, for IS classes [for example, 4, 34]. In order to construct a lightweight ISDM selection for SMEs, design principles are a promising approach that considers solution objectives as well as problem context - but pioneering work is needed to apply existing design principle-related knowledge for constructing a method, not a class of IS.

\section{ISDM selection and management method}

As mentioned, the knowledge areas of business development and ISD rarely overlap. On the other hand, system development should be based on business needs to advance strategic business development. Thus, our rationale is that the ISD service buyer may not be knowledgeable enough to select and/or customize a method. Our aim is to bridge the knowledge gap using three theoretically justified models or steps (as illustrated in Figure 2): the business context evaluation model (3.1), the ISDM selection model (3.2) and the ISDM management model (3.3).

As the proposed method is aimed at SMEs, it should be lightweight and dynamic. The rationale for the SMEs needing such a method in the first place was that they lack the capabilities to evaluate the development context in general and the suitability of the proposed method (the software developer often proposes the ISDM). Thus, the ISDM selection and

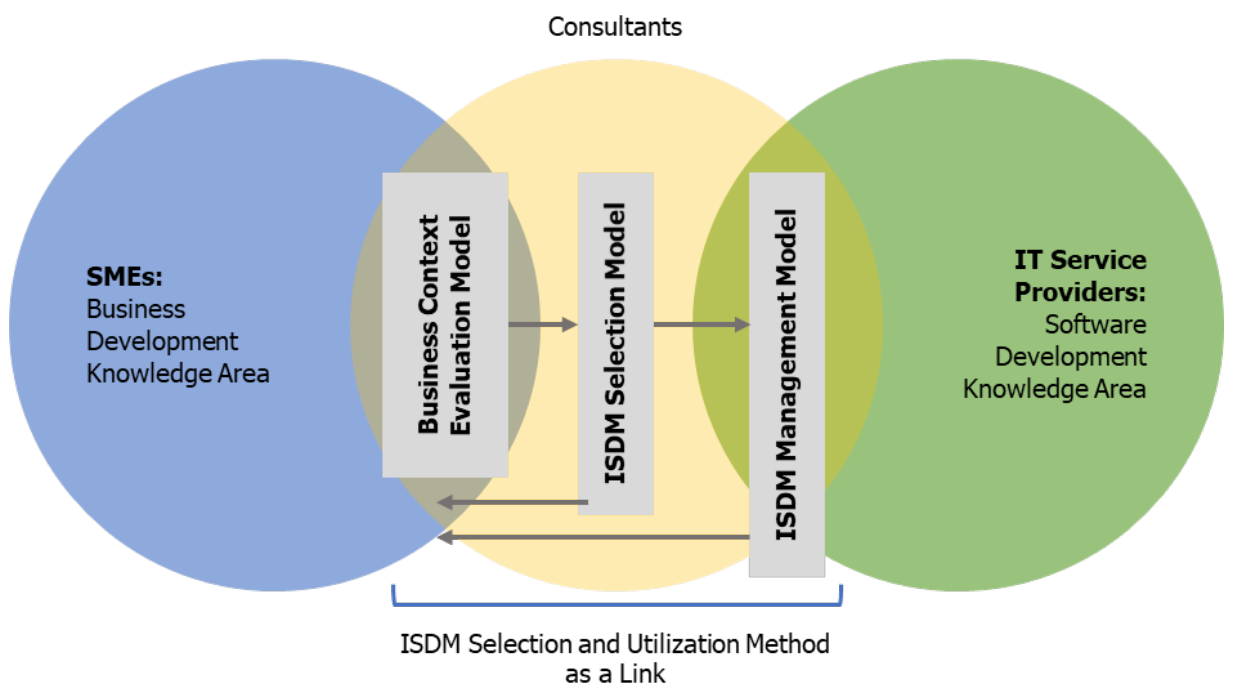

Figure 2. Method as a link between the distinct knowledge areas 
management method should be practical and simple enough.

The timing of the decision making is critical. It is not possible to decide on every aspect at once, so a stepwise approach (Figure 3) is proposed. From the three steps presented, the first two must be taken before the IS developer is selected, or even before a request for proposals is sent out. In practice, this means that the client or buying organization (SME) decides on an ISDM before discussing details of the project with the IS developer. In most cases, this requires help, and steps 1 and 2 aim to provide that. Step 3 is intended for later use during the realization of the development project.
ISD project and business context characteristics with ISDM characteristics, the selection model will lead to the most suitable (combination of) method components based on the existing models, standards and tools that are available for the project. The project team and its characteristics are also used as parameters. The input for the selection should be expressed on a sliding scale as in Lagstedt and Dahlberg [29], rather than just high or low.

Naturally, in our lightweight selection model the aim is not to automate the calculation of all the parameters, but rather to provide models that support systematic reflection and inform decision making. The context-aware selection continues from the results of

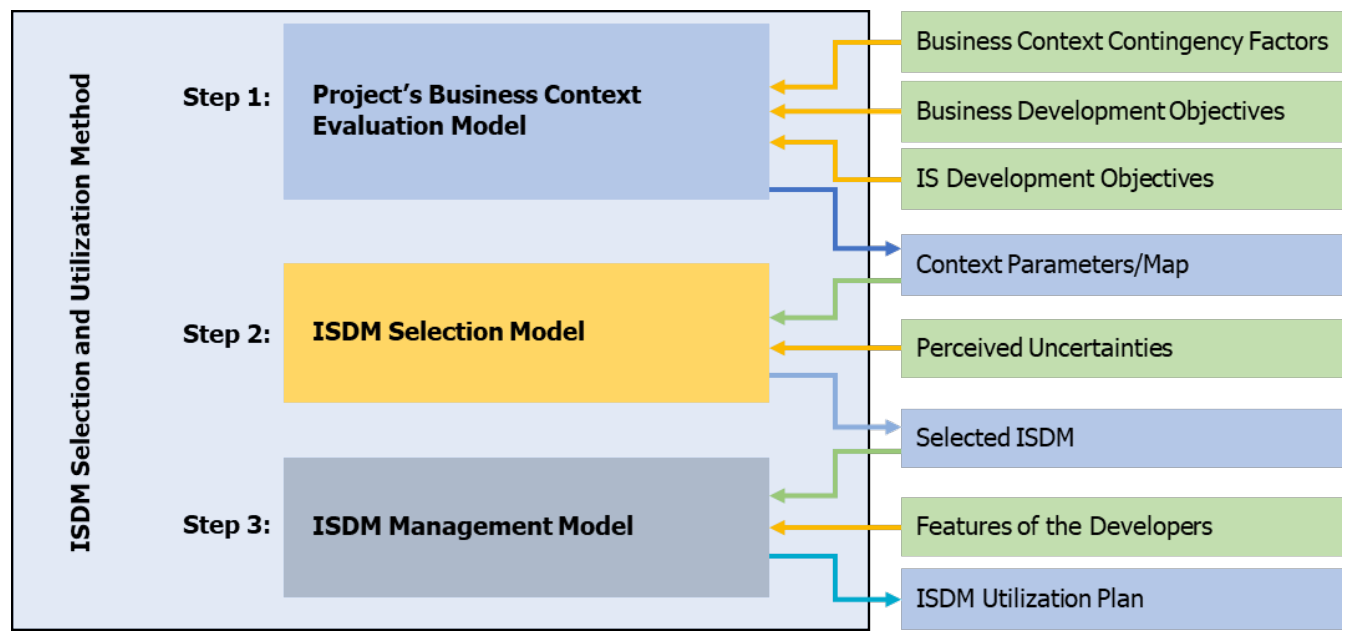

Figure 3. Proposed ISDM selection and utilization method

\subsection{Business context evaluation model}

The business context needs to be evaluated to understand the needs of the business development project associated with the ISD project. The evaluation model analyzes the situation from three angles: 1) ISD objectives, 2) business process development objectives and 3) factors from the project's business context. Here, we use the factors adapted from Kiselev et al. [27]: terrain, dynamics, complexity, commitment and ability to act (Table 1). By assessing the factors, the nature of the business development and ISD projects together becomes clearer, and the findings constitute a base for selecting an appropriate ISDM.

\subsection{ISDM selection model}

As parameters for a selection, the ISDM selection model uses (a) the output of the context evaluation model, (b) analysis of the business situation and business execution, and (c) uncertainty analysis of the business development outcomes [29]. By matching the the first step of the proposed method. Actor making the selection is provided with mapping of factors and ISDMs, from which to select the most suitable route.

In addition to proposing a method (or certain combination of method components), the model includes documentation of the selection causes. These causes are used in the utilization phase to manage the ISD process and explain the selection that was made.

\subsection{ISDM management model}

From a SME perspective, the relevant method components [26, 36] are derived from existing standards, frameworks, models and best practices. Technological rules (or, in more complex cases, situational configuration rules) combine these widely context-agnostic components with the situationspecific needs of the project.

The management model will use the outcomes of the business context evaluation and ISDM selection models as a starting point - that is, the method is already selected, and the development context analyzed 
and understood. Here, in addition to the selected (combination of) ISDM, the factors leading to the selection affect the management roadmap.

Based on the ISDM management model, different methods and tools are used in different parts and phases of the ISD project, reflecting the characteristics of the development team and the context. The basic guidelines for managing ("governing”) an ISD project and aligning between the three stakeholders (SME, IS developer and consultancy) can be adapted from the general guidelines presented by Kiselev et al. [27]

Another benefit of using the management model to draw a roadmap for ISDM use within an ISD project is the forced use of standards. As mentioned, the developers can be unaware of the existing standards [30, 31], which hampers successful IS development.

With the management model SMEs can manage both the development project and the outcome of the development, without having detailed-level knowledge of development methods and standards.

\section{Initial evidence from pilot projects}

The main components of our proposed method have been initially tested in some of the cases we have analyzed in earlier research. Our plan is to collect further evidence in additional action design research project(s), particularly aiming at integrating method components in projects which span complete digital transformation journeys.

For the context evaluation phase of the method, Kiselev et al. [27] report how, under the label "triage guidelines," the proposed context evaluation has been implemented partially by additions to a widely used project management standard and partly by certain governance recommendations. As these standard additions and guidelines have been in effect for two years now, "local" empirical validation evidence of context-awareness utility is now available but has not yet been integrated with method selection.

Some preliminary research has already been done related to ISDM selection model as well. The existing ISDM selection literature was studied in a systematic literature review from autumn 2015 through spring 2016. Based on the findings of the literature review, the experts (consultants, $n=31$ ) working along the borderline between IS development clients and developers were interviewed in 2016. Both the literature review and the interviews confirmed the notion that 1) there is a need for a selection model, 2) the recommendations published before 2015 are more or less outdated and 3) the context of the development should be taken into account when ISDM selection is done. In the interviews the Lagstedt and Dahlberg model (Figure 1) was considered a good start for a business context-aware ISDM selection model.

For the ISDM management model, Kiselev et al. [27] report how, under the label "ability guidelines," digitalization governance (and not digitalization project management which is usually left to the consultant and/or the software developer) is supported by providing clear and simple steering guidelines and establishing quality control. A short project sponsor manual has been published that is frequently used in digitalization projects to guide project sponsors and steering committees in defining their tasks, competencies and responsibilities. The manual also guides steering committee meetings through standard agenda items and defines compulsory project checkpoints - always from a project sponsor and not a project management perspective.

In the coming research projects, our aim is to focus on the connections the business context must ISDM selection and management. Furthermore, the research will clarify the use cases of the integrated approach from the perspective of the main actors (SMEs, consultants, and IT service providers).

\section{Discussion and conclusions}

The contradiction between the growing importance of IS for almost any type of business and the lagging ability to develop IS or even buy ISD services sets an interesting backdrop for our conceptual study. It is understandable that organizations whose core business is anywhere other than ISD do not master the skills needed to select an ISDM for ISD projects. Even more, it is understandable and explicable that SMEs, which often have limited capabilities and financial possibilities for developing new digital business and digitalizing existing business models, are lagging in digitalization [22]. It is desirable that when the SMEs are taking their smaller steps towards digitalized business, these steps are more often successful than unsuccessful. Tools supporting their decision making in ISD projects can help with this desire.

Until recently, contemporary academic research has widely neglected the business context in ISDM selection, only providing at least partly outdated options that mainly focus on the project characteristics [17]. Considering the changing circumstances (e.g., digitalization, electronic business models) in which business is done, an updated ISDM selection method is needed. As we have mentioned, the changing circumstances are even more real for SMEs, as their business environments and needs are so diverse. No one development service provider can offer solutions for these diverse needs, not even to most of them, as there are no standard solutions. 
Research has provided models for analyzing the business context of the ISD project [27, 47], simplified models to select the ISDM [29] and ways of configuring the selected methods to suit the project's needs [e.g., 45]. In this conceptual research, we have composed a three-step method to select the ISDM, starting from the business context analysis and not making all the decisions at once, but carefully considering the relevant factors and objectives related to the ISD project and its business context. Moreover, we propose that the documentation of the ISDM selection process and the existing ISD standards, tools and methods should be utilized when using the selected ISDM. By adding the proposed ISDM selection method to the requirements specification phase of the ISD project, SMEs can be more prepared and knowledgeable of the consequences the selected (combination of) ISDM has on the overall ISD project. The selection route will be documented and thus guide the actual usage of the ISDM.

In this conceptual paper we have laid out the framework for ISDM selection and utilization method. As noted, the different components (models) of the method have already been examined in previous studies giving preliminary evidence for the business context evaluation model, ISDM selection model and ISDM management model. This lays the groundwork for our forthcoming research project, in which we aim to integrate these different models into a usable ISDM selection and utilization method. In forthcoming research projects, we will also further develop the individual models and test the developed ISDM selection and utilization method in case studies.

In conclusion, and as an answer to the research question of this paper ("How ISDM decision-making can be supported for SMEs"), we state that the business development context should be carefully analyzed using the factors of terrain, dynamics, complexity, commitment and ability to act [27] together with the certain uncertainties related to the project [29]. Using the results of these analyses as parameters, a context-aware ISDM can be constructed to suit the specific needs of the project and business development, and an appropriate utilization and management model can be proposed that guides SMEs and providers toward better-aligned solution development.

\section{References}

[1] Ahimbisibwe, A., R.Y. Cavana, and U. Daellenbach, “A contingency fit model of critical success factors for software development projects A comparison of agile and traditional plan-based methodologies", Journal of Enterprise Information Management 28(1), 2015, pp. 7-33.
[2] van Aken, J.E., "Management research based on the paradigm of the design sciences: the quest for field-tested and grounded technological rules”, Journal of management studies 41(2), 2004, pp. 219-246.

[3] Bharadwaj, A., O. El Sawy, P. Pavlou, and N. Venkatraman, "Digital Business Strategy: Toward a Next Generation of Insights”, MIS Quarterly 37(June), 2013, pp. 471-482.

[4] Blaschke, M., U. Riss, K. Haki, and S. Aier, "Design principles for digital value co-creation networks: a servicedominant logic perspective”, Electronic Markets 29(3), 2019, pp. 443-472.

[5] Boehm, B.W., and R. Turner, Balancing agility and discipline: A guide for the perplexed, Addison-Wesley Professional, 2004.

[6] Borg, M., J. Wernberg, T. Olsson, U. Franke, and M. Andersson, "Illuminating a Blind Spot in Digitalization Software Development in Sweden's Private and Public Sector", IEEE/ACM 42nd International Conference on Software Engineering Workshops (ICSEW'20), (2020). [7] Bouwman, H., S. Nikou, and M. de Reuver, "Digitalization, business models, and SMEs: How do business model innovation practices improve performance of digitalizing SMEs?”, Telecommunications Policy 43(9), 2019, pp. 101828.

[8] Brinkkemper, S., “The Software Product Management Workbench View project A theory building study of enterprise architecture practices and benefits View project Method engineering: engineering of information systems development methods and tools”, Elsevier 38, 1996, pp. 275280.

[9] Bucher, T., M. Klesse, S. Kurpjuweit, and R. Winter, "Situational method engineering: On the differentiation of 'Context' and 'Project Type'”, IFIP International Federation for Information Processing, Springer, Boston, MA (2007), 33-48.

[10] Burns, R.N., and A.R. Dennis, "Selecting the appropriate application development methodology”, ACM Sigmis Database(613), 1985.

[11] Canziani, B.F., and D.H.B. Welsh, "Website quality for SME wineries: measurement insights”, Journal of Hospitality and Tourism Technology 7(3), 2016, pp. 266-280.

[12] Chandra, L., S. Seidel, and S. Gregor, "Prescriptive knowledge in IS research: Conceptualizing design principles in terms of materiality, action, and boundary conditions", 2015 48th Hawaii International Conference on System Sciences, (2015), 4039-4048.

[13] Cooper, R.G., "Stage-Gate Systems : A New Tool for Managing New Products”, Business Horizons(May-June), 1990.

[14] Cunningham, W., "Experience Report- The WyCash Portfolio Management System”, ACM SIGPLAN OOPS Messenger 4(2), 1992, pp. 29-30.

[15] Dahlberg, T., and H. Kivijarvi, “Towards an integrative, multilevel theory for managing the direct and indirect impacts of IT project success factors”, Proceedings of the Annual Hawaii International Conference on System Sciences, IEEE Computer Society (2016), 4971-4980.

[16] Dahlberg, T., and A. Lagstedt, “There Is Still No 'Fit for All' IS Development Method: Business Development Context and IS Development Characteristics Need to Match”, 
Proceedings of the 51st Hawaii International Conference on System Sciences, Hawaii International Conference on System Sciences (2018).

[17] Dahlberg, T., and A. Lagstedt, "The Usefulness of the Recommendations Regarding the Information System Development Method Selection during the Era of Digitalization", Proceedings of the 52nd Hawaii International Conference on System Sciences 2019, (2019). [18] Denyer, D., D. Tranfield, and J.E. van Aken, "Developing Design Propositions through Research Synthesis”, Organization studies 29(3), 2008, pp. 393-413. [19] Fitzgerald, B., "An empirical investigation into the adoption of systems development methodologies", Information and Management 34(6), 1998, pp. 317-328. [20] Ghobakhloo, M., M. Sadegh Sabouri, T. Sai Hong, and N. Zulkifli, "Information Technology Adoption in Small and Medium-sized Enterprises; An Appraisal of Two Decades Literature”, Interdisciplinary Journal of Research in Business 1(7), 2011, pp. 53-80.

[21] Gregor, S., L.C. Kruse, and S. Seidel, "The Anatomy of a Design Principle”, Journal of the Association for Information Systems, 2020.

[22] Gruber, H., "Proposals for a digital industrial policy for Europe”, Telecommunications Policy 43(2), 2019, pp. 116127.

[23] Gu, V.C., J.J. Hoffman, Q. Cao, and M.J. Schniederjans, "The effects of organizational culture and environmental pressures on IT project performance: A moderation perspective", International Journal of Project Management 32(7), 2014, pp. 1170-1181.

[24] Hansen, S., and K. Lyytinen, "Challenges in contemporary requirements practice”, Proceedings of the Annual Hawaii International Conference on System Sciences, (2010).

[25] Howell, D., C. Windahl, and R. Seidel, “A project contingency framework based on uncertainty and its consequences”, International Journal of Project

Management 28(3), 2010, pp. 256-264.

[26] Karlsson, F., and P.J. Ågerfalk, "Method configuration: Adapting to situational characteristics while creating reusable assets”, Information and Software Technology 46(9), 2004, pp. 619-633.

[27] Kiselev, C., R. Winter, and P. Rohner, "Project Success Requires Context-Aware Governance”, MIS Quarterly Executive 19(3), 2020, pp. 199-211.

[28] Lagstedt, A., and T. Dahlberg, "Understanding the Rarity of ISD Method Selection - Bounded Rationality and Functional Stupidity”, Pacific Asia Conference on Information Systems, (2018), 1-14.

[29] Lagstedt, A., and T. Dahlberg, “A Contingency Theory Motivated Framework to Select Information System Development Methods”, PACIS 2018 Proceedings, 2018. [30] Laporte, C.Y., M. Munoz, J. Mejia Miranda, and R. V. Oconnor, “Applying Software Engineering Standards in Very Small Entities: From Startups to Grownups”, IEEE Software 35(1), 2017, pp. 99-103.

[31] Laporte, C.Y., and R. V. O’Connor, "Systems and Software Engineering Standards for Very Small Entities: Accomplishments and Overview”, Computer 49(8), 2016, pp. 84-87.
[32] Larman, C., and V.R. Basili, "Iterative and incremental development: A brief history”, Computer 36(6), 2003, pp. 47-56.

[33] Marinho, M., J. Noll, I. Richardson, and S. Beecham, "Plan-Driven Approaches Are Alive and Kicking in Agile Global Software Development”, International Symposium on Empirical Software Engineering and Measurement, IEEE Computer Society (2019).

[34] Marques, R., G. Costa, M. Mira da Silva, and P. Gonçalves, "A survey of failures in the software development process", Proceedings of the 25th European Conference on Information Systems, ECIS 2017, (2017), 2445-2459.

[35] Meth, H., B. Mueller, and A. Maedche, "Designing a requirement mining system”, Journal of the Association for Information Systems 16(9), 2015, pp. 799.

[36] Mirbel, I., and J. Ralyté, "Situational method engineering: Combining assembly-based and roadmap-driven approaches”, Requirements Engineering 11(1), 2006, pp. 5878.

[37] Moe, N.B., A. Aurum, and T. Dybå, "Challenges of shared decision-making: A multiple case study of agile software development”, Information and Software Technology 54(8), 2012, pp. 853-865.

[38] Noll, J., and S. Beecham, "How Agile Is Hybrid Agile? An Analysis of the HELENA Data”, Lecture Notes in Computer Science (including subseries Lecture Notes in Artificial Intelligence and Lecture Notes in Bioinformatics), Springer (2019), 341-349.

[39] Savolainen, P., J.J. Ahonen, and I. Richardson, “When did your project start? - The software supplier's

perspective”, Journal of Systems and Software 104, 2015, pp. 32-40.

[40] Sommerville, I., Software Engineering, AddisonWesley, Boston, Massachusetts, 2011.

[41] Standish Group, “CHAOS Report 2015”, The Standish Group International, Inc., 2015, pp. 13.

[42] Taylor, H., "Outsourced IT Projects from the Vendor Perspective: Different Goals, Different Risks”, Journal of Global Information Management 15(2), 2007, pp. 1-27.

[43] Theocharis, G., M. Kuhrmann, J. Münch, and P.

Diebold, "Is Water-Scrum-Fall Reality? On the Use of Agile and Traditional Development Practices", Lecture Notes in Computer Science 9459, 2015, pp. 149-166.

[44] Truex, D., R. Baskerville, and J. Travis, “Amethodical systems development: The deferred meaning of systems development methods", Accounting, Management and Information Technologies 10(1), 2000, pp. 53-79.

[45] De Weger, M.K., and H.M. Franken, “A situational approach to design strategies”, Software Quality Journal 6(3), 1997, pp. 181-194.

[46] Winter, R., "Construction of situational information systems management methods”, International Journal of Information System Modeling and Design, (2012), 67-85. [47] Winter, R., P. Rohner, and C. Kiselev, "Mission Impossible? Exploring the Limits of Managing Large IT Projects and Ways to Cross the Line", Proceedings of the 52nd Hawaii International Conference on System Sciences, Hawaii International Conference on System Sciences (2019). 